\title{
Practical Aspects of Instantaneous Power Control of Induction Machines
}

\author{
R.E. Betz ${ }^{\dagger}$, S.J. Henriksen*, B.J. Cook ${ }^{\ddagger}$, T. Summers ${ }^{\diamond}$ \\ Department of Electrical and Computer Engineering \\ The University of Newcastle, Australia \\ FAX: +61-2-49-6993 \\ Email: ${ }^{\dagger}$ reb@ee.newcastle.edu.au; *eesjh@ee.newcastle.edu.au; \\ ${ }^{\ddagger}$ brian@ee.newcastle.edu.au; ^terry@ee.newcastle.edu.au
}

\begin{abstract}
High performance induction machine control is usually implemented using field oriented control (FOC) or direct torque control (DTC). These strategies have their strengths and weaknesses. The concept of instantaneous power control (IPC) has been used in power compensators and to control the voltage on the DC link of inverters, but it is a new concept for the control of induction machines. The idea is that by controlling the instantaneous real and imaginary power into the machine one can control the torque and flux of the machine.

This paper outlines the development of the algorithm, and then focuses on some practical issues associated with the implementation of the algorithm.
\end{abstract}

\section{INTRODUCTION}

$\mathrm{T}$ HE induction machine is still the mainstream electrical machine in use today. Consequently there is still considerable research effort being expended to understand how to reliably use this machine in high performance applications that were traditionally the domain of the DC machine.

The first algorithm that was able to achieve high performance control of induction machines was field oriented control (FOC), invented some 30 years ago [1]. Since this time it has become a common control technique. A second algorithm, known as direct torque control (DTC) $)^{1}$, has become popular since the late 1980s. Both these algorithms have their strengths and weaknesses. We shall briefly review them, and then compare their attributes to the instantaneous power control (IPC) algorithm, which is the subject of this paper.

The FOC technique, as the name implies, relies on spatial knowledge of either the stator, rotor or magnetising field vector to position the reference frame for the control calculations. In practice the stator or rotor fields are generally used. The alignment of the control equations reference frame with one of these fields allows the control of the torque to be decoupled from the flux. If there is an error in the position of the frame then coupling between the flux and torque axes is introduced, resulting in torque oscillations during transients. Obtaining an accurate position of the flux vector turns out to be an inherently parameter sensitive operation. For example, in indirect FOC, the rotor resistance is the main parameter determining the position of the rotor flux vector. During machine operation this

\footnotetext{
${ }^{1}$ A closely related algorithm is Direct Self Control(DSC) [2].
}

parameter value changes substantially. Nevertheless, considerable research has been carried out on the on-line determination of rotor resistance, and successful drives are being built using indirect FOC. The other form of FOC is direct FOC. This relies on an accurate flux model of the machine, which is parameter dependent.

Operation in a rotating reference frame made FOC a relatively complex control technique to implement, especially 20 to 30 years ago when microprocessors had difficulty dealing with coordinate transformations. On the positive side the synchronous nature of the reference frames results in DC values for the currents and voltages in steady state. This allows conventional PI current control loops to be used. The use of a symmetric PWM algorithm means that the switching frequency and output harmonics are well defined.

DTC, as the name implies, is a technique for directly controlling the torque and flux of the machine, without the intermediate step of using a current controller. The DTC algorithm operates in a stationary reference frame, therefore frame conversions are not required. DTC works by detecting the field position and magnitude from measurements of the terminal voltage of the machine. Based on this, and the torque error, a voltage vector is chosen from a table to move both the flux and the torque in the directions towards their desired values. DTC can be implemented using analogue techniques (which usually results in a variable switching rate), or alternative via a table driven approach that is very amenable to implementation in a processor.

One of the most attractive features of DTC is that in its pure form it is not parameter dependent - it appears to derive the control from measurements only. Another feature is the simplicity of not having a current controller. However, in order to achieve satisfactory low speed performance, and minimise torque and flux ripple, the algorithm becomes parameter dependent. The variable switching frequency, due to the hysteresis property of the current control, is not satisfactory in many applications, especially those in the medium to high power range. Considerable research has been undertaken in order to overcome the deficiencies of the DTC approach, but most solutions usually involve the algorithm becoming more machine param- 
eter dependent [3].

This paper considers an alternative to the above two algorithms which will be called instantaneous power control (IPC). The basic control strategy is fundamentally different as compared to FOC and DTC in that the control variables are the instantaneous real and imaginary powers flowing into the machine. In many respects one can think of the IPC strategy as being a dual of the conventional techniques.

The crux of the idea behind IPC is that by controlling the real power one can control the torque produced by the machine, and the imaginary power can control the flux in the machine. In some applications, such as static compensators, control of the imaginary power may be a control objective in its own right (since the imaginary power is closely related to the reactive power) [4]. The concept of instantaneous real and imaginary power control is not new $[4,5]$, but the utilisation of this principle to control induction machines is.

The initial motivation for this technique of induction machine control was the perceived simplicity of having decoupled control of torque and flux via real and imaginary power. A superficial consideration of the algorithm leads one to believe that this can be achieved via a controller without machine parameter dependency. Unfortunately, the IPC algorithm has inherent cross-coupling between the real and imaginary powers with respect to controlling torque and flux. The decoupling equations involve machine parameters. If the control objective is simply the real and imaginary powers themselves, then this cross-coupling does not exist.

IPC can be considered to have characteristics of both FOC and DTC. Like FOC it utilises a current controller and a more or less conventional PWM algorithm. This ensures that it has the predictable switching and spectrum characteristics of FOC. Similarly to DTC it operates in a stationary reference frame and utilises voltage estimates. The reliance on voltage estimates means that this algorithm cannot operate at zero speed. ${ }^{2}$

In the remainder of this paper we shall outline the IPC algorithm, and then discuss some important implementation issues.

\section{Algorithm Outline}

The IPC algorithm has already been published [6], and therefore we shall only briefly outline it here for completeness and immediate reference. More detail can be obtained in [7].

The IPC algorithm for an induction machine has three main components as shown in Fig. 1: the power reference generator (PRG), the current reference generator (CRG), and the predictive current controller (PCC).

The inputs to the PRG are shown as ref, $m$ and ref, which are the desired output torque, the measured shaft speed, and the desired flux. Also going into this block are the back-emf voltages. These cannot be measured, and must be estimated. The torque and flux come from an outside reference generator

\footnotetext{
${ }^{2}$ In addition zero speed operation means that the power required to produce an arbitrary torque is always zero.
}

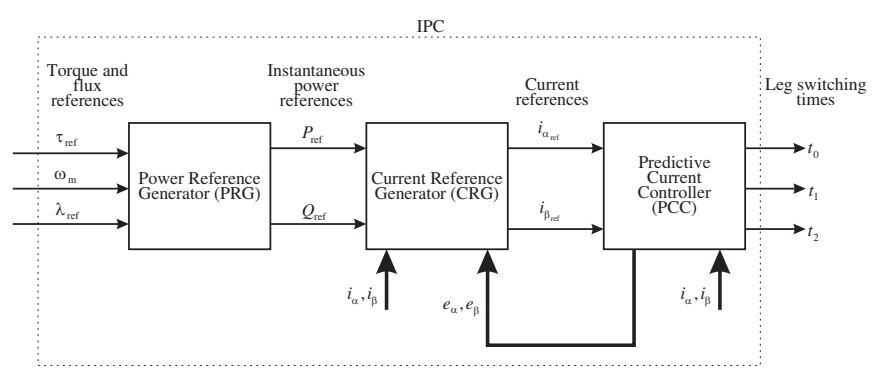

Fig. 1. Block diagram of IPC for an induction machine.

(e.g. from a speed control loop). The $m$ value is required to generate the power references from ref.

The CRG section of the controller is relatively straight forward, being directly based on the complex power expression:

$$
{ }_{-}{ }_{--}^{*}
$$

which can be written in terms of the two phase stationary frame currents and voltages as $[6,7]$ :

$$
\begin{aligned}
& P=\quad+ \\
& Q=
\end{aligned}
$$

It is well known that an induction machine can be represented with good accuracy by a voltage source behind a leakage inductance [8-10]. The voltage source is essentially a slowly changing fundamental frequency sinusoidal source, even when the machine is being driven by a PWM voltage source. The high frequency components of the PWM waveform are supported across the leakage inductance. Therefore the instantaneous power absorbed by the machine (i.e. the real power and imaginary power) is related to the instantaneous current times the internal voltage source. This then allows us to write (2) and (3) as:

$$
\begin{aligned}
& P=\quad+ \\
& Q=
\end{aligned}
$$

where represent the two phase equivalent internal backemfs.

Remark 1: The instantaneous real power $P$ is the sum of the power absorbed by the resistances of the machine, the iron losses, transient field storage, and the output shaft power. Similarly the imaginary power $Q$ is related to the magnitude and rotational speed of the flux vector. Note that the rotational speed of the flux vector is related to the slip frequency and shaft speed of the machine, The slip frequency is related to the torque produced and real power absorbed by the machine. $\diamond$

Remark 2: Remark 1 demonstrates the cross-coupling relationship between real and imaginary power when torque and flux control is required. Notice that torque demands will influence the imaginary power, and similarly changes in imaginary power will result in real power flows due to changes in the stored field energy of the machine. Also note that even for a 


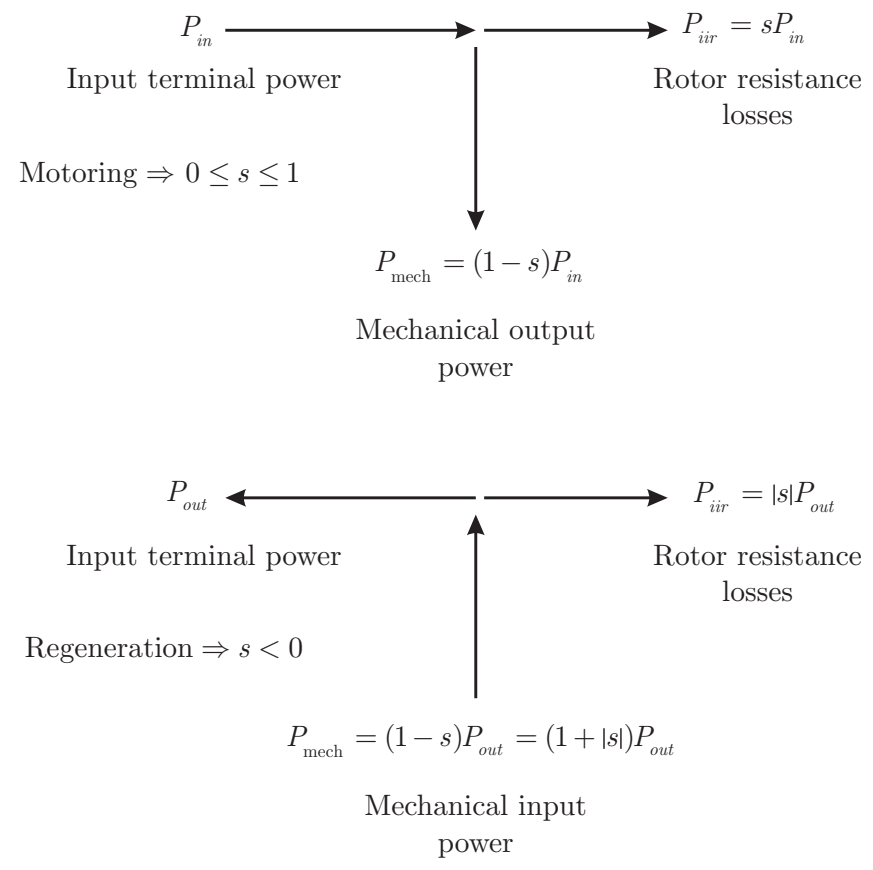

Fig. 2. Approximate real power flows in an induction machine with constant flux.

constant flux in the machine, the imaginary power for the machine will not be a constant - it is effectively a function of the fundamental frequency of the machine supply. $\diamond$

From (4) and (5) one can see that for any arbitrary $P_{\text {ref }}$ and $Q_{\text {ref }}$, and assuming that can be estimated, one can simultaneously solve for the reference currents:

$$
\begin{aligned}
\text { ref } & =\frac{P_{\text {ref }} Q_{\text {ref }}}{2+2} \\
\text { ref } & =\frac{P_{\text {ref }}+Q_{\text {ref }}}{2+{ }^{2}}
\end{aligned}
$$

Remark 3: One immediate observation that can be made from (6) and (7) is that they cannot be solved if $==0$. Furthermore the calculation is ill-conditioned if these voltages are small. This would occur at low shaft speeds. $\diamond$

The current references generated by (6) and (7) are then passed to a predictive current controller (PCC) $[11,12]$. A PCC is used because of its natural digital implementation, and also because it inherently estimates the required back-emf voltages required for the CRG. We shall look at some practical issues in relation to the $\mathrm{PCC}$ later in this paper.

The non-obvious part of the IPC algorithm is the development of the $P_{\text {ref }}$ and $Q_{\text {ref }}$ references. As mentioned in Remark 1, there is cross coupling between $P$ and $Q$. Let us firstly consider the real power reference, under the assumption that the flux magnitude is being held constant. The simple way to generate this reference is to use the expression $P_{\text {ref }}=$ ref $m$. However, this expression leads to significant output torque errors. Fig. 2 shows the approximate real power flows in an in- duction machine ${ }^{3}$. Notice that under motoring conditions the the real power can flow into the shaft output power, but also into rotor resistance losses. This results in an error between the desired output power and the actual output power.

Under regeneration the output power errors become much larger. In this situation the desired $P_{\text {ref }}$ can be obtained at the output, but the $P_{\text {mech }}$ can have an arbitrary value due to the fact that the magnitude of the slip ( ) can become very large. ${ }^{4}$ When this happens large amounts of power can be dumped into the rotor resistance from the shaft output. Consequently, there would be a very high decelerating shaft torque. Fortunately, controlling $Q$ implicitly places a limit on the slip under these conditions. $^{5}$

If we assume for the moment that the machine slip is known, then $P_{\text {ref }}$ can be compensated as follows:

$$
P_{\text {ref }}=\frac{P_{\text {mech }}}{1 \quad \text { comp }}
$$

where comp is the compensating slip.

For an induction machine we can write [6]:

$$
\left.Q_{\text {ref }} \quad m r_{\text {ref }}\right|_{-} \mid=m r_{\text {ref }} \quad m_{\text {ref }}
$$

where $m r_{\text {ref }} \triangleq$ the reference magnetising current.

Using $=m+s l$, where $s l \triangleq$ the slip frequency, one can, after a little manipulation, write $[6,7]$ :

$$
\text { comp }=1+\frac{r m{\stackrel{m}{m} r_{\text {ref }}}^{2}}{Q_{\text {ref }}}
$$

The other reference to be found is $Q_{\text {ref. }}$ Using expressions for torque and slip frequency from the FOC equations [6,7] one can develop the following expression for the imaginary power reference:

$$
Q_{\mathrm{ref}}=m_{m}^{2} r_{\mathrm{ref}} r+\frac{2}{3 \text { ref }}
$$

where $T_{r} \triangleq$ the rotor time constant, and $\triangleq$ the machine pole pairs.

Remark 4: One can see from (11) that the imaginary power reference is a function of both the shaft electrical angular velocity, and the torque produced by the machine. The latter is a function of the slip of the machine. Therefore for a constant $m_{\text {ref }}=m m r_{\text {ref }}, Q_{\text {ref }}$ is a dynamically varying value. Note that if there is a sudden desired torque transient then $Q_{\text {ref }}$ will undergo a corresponding step change. $\diamond$

Fig. 3 shows a detailed diagram of an IPC based speed controller for an induction machine.

\footnotetext{
${ }^{3}$ This diagram is a simple representation of the power flows. For example, one must have a non-zero slip for a non-zero $P_{\text {mech }}$, but this is not represented in Fig. 2.

${ }^{4}$ In the motoring case the slip is implicitly limited to 1.

${ }^{5}$ These effects were observed in the simulations carried out.
} 


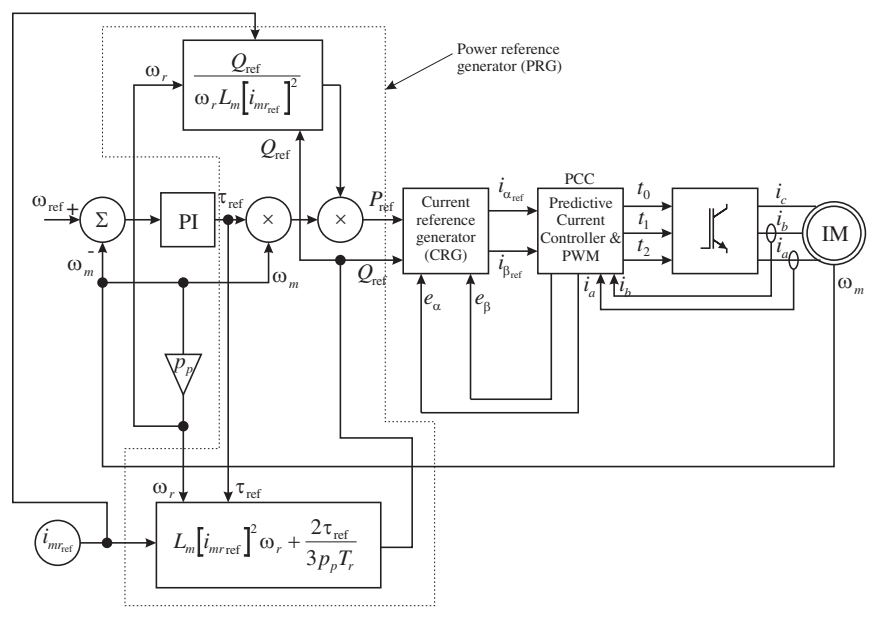

Fig. 3. Detailed block diagram of an IPC based speed controller.

\section{PRACTICAL IsSUeS}

The performance of IPC under ideal conditions was shown to be satisfactory in [6]. However, when implemented there are a number of practical issues that impinge on the performance of the controller. The issues that will be examined in this paper are:

1. Sensitivity to parameter accuracy in the CRG.

2. Parameter issues in the PCC.

3. Dead-time influence on the performance of the PCC and IPC.

\section{A. Parameter Sensitivity}

The generation of ref and ref in the CRG via (6) and (7) is parameter independent (at least in a direct sense), but the generation of $P_{\text {ref }}$ and $Q_{\text {ref }}$ in the PRG using (8) and (11) is certainly not. Therefore a valid question to ask is; "what is the effect of inaccurate parameter knowledge on the performance of the algorithm?". In order to allow some relative measure of the performance of IPC it shall be compared with the performance of an indirect FOC under the same conditions.

The simulation results to be presented in this section are for an induction machine with the parameters shown in Table I. The plots for accurate parameters appear in [6] and will not be repeated here. We shall consider the case where there is a 50\% error in the rotor time constant - specifically we are assuming that the actual machine $R_{r}$ parameter varies by $+50 \%,{ }^{6}$ which in turn means that the rotor time constant varies by $50 \%$. Therefore the time constant being used in the controller is $50 \%$ larger than the actual machine time constant.

The following results have been generated using the Saber ${ }^{\circledR}$ simulation package. The desired flux magnitude is 0.72 Weber, and the speed controller is demanding a series of step changes in speed $-30 \mathrm{rad} / \mathrm{sec}, 100 \mathrm{rad} / \mathrm{sec}, 50 \mathrm{rad} / \mathrm{sec}$. Note that the machine is started using a FOC, and at $250 \mathrm{msec}$ the IPC switches in. Nothing appears to happen for the first $200 \mathrm{msec}$ because

\footnotetext{
${ }^{6}$ This is the most common situation since $R_{r}$ increases in value with rotor heating.
}

\begin{tabular}{|c|c|}
\hline Parameter & Value \\
\hline \hline$m$ & $0.0693 \mathrm{H}$ \\
\hline$r$ & $0.0713 \mathrm{H}$ \\
\hline$s$ & $0.0713 \mathrm{H}$ \\
\hline$R_{r}$ & $0816 \Omega$ \\
\hline$R_{s}$ & $0435 \Omega$ \\
\hline Poles & 4 \\
\hline$J$ & $0.089 \mathrm{kgm}^{2}$ \\
\hline
\end{tabular}

TABLE I

MACHINE PARAMETERS

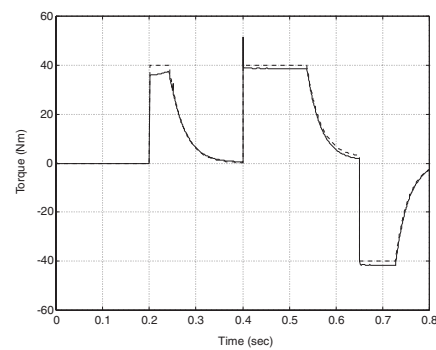

(a) Torque

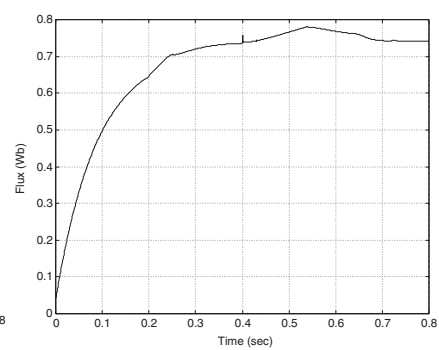

(b) Flux
Fig. 4. IPC torque and flux with $50 \% T_{r}$ error.

the demanded torque is zero whilst the flux is building up in the machine.

Remark 5: The most striking feature of Figs. 4 and 5 is that the torque and the flux in Fig. 4 is reasonably well controlled compared to the plots in Fig. 5. In fact the FOC virtually looses control of the flux. If plots are generated for the dual situation (i.e. $T_{r}$ in the controller is $50 \%$ less than the actual rotor time constant) a similar result applies - the IPC algorithm has far better performance compared to FOC. It should be noted however, the the performance of IPC is slightly worse in this situation compared to the $T_{r} 50 \%$ larger scenario. $\diamond$

Remark 6: The parameter insensitivity of IPC in relation to the parameters in the reference generation decoupling network bode well for the practical implementation of the algorithm. $\diamond$

The other parameters that appear in the IPC algorithm are situated in the PCC. PCC algorithms are largely parameter independent, since they attempt to lump all the parameters into the back-emf voltage of the voltage behind leakage inductance

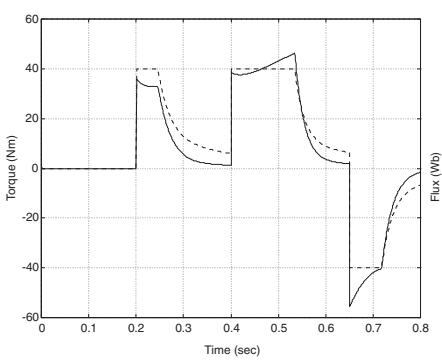

(a) Torque

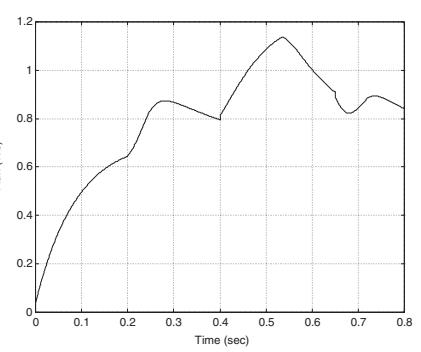

(b) Flux
Fig. 5. FOC torque and flux with 50\% $T_{r}$ error. 
model. Therefore two factors are important in the operation of this algorithm - the accuracy of the back-emf estimates, and the sensitivity of the algorithm to knowledge of the leakage inductance. Let us firstly consider the leakage inductance issue.

The PCC controller equations for each of the two phases, in discrete form, can be shown to be [10-13]:

$$
\begin{aligned}
{ }_{1} & =\frac{{ }_{l} \Delta}{T}\left({ }_{1}{ }_{1}{ }_{1}\right)+\begin{array}{r}
\text { pred } \\
1
\end{array} \\
{ }_{1} & ={ }_{1} \frac{{ }_{l} \Delta}{T}\left(\begin{array}{ll}
1
\end{array}\right)
\end{aligned}
$$

where $\quad l$ is the actual machine leakage, and ${ }_{l} \Delta$ is the estimated leakage, $\Delta$ being a multiplicative estimation error. ${ }^{7}$

If we assume that ${ }_{1}^{\text {pred }}={ }^{\text {est }}$ then we can write (12) and (13) as follows:

$$
1=\frac{{ }_{l} \Delta}{T}\left({ }_{1}^{\operatorname{ref}} \quad\right) \quad \frac{{ }_{l} \Delta}{T}(\quad 1)
$$

where is introduced to account for the mid-point and end point control forms of the control algorithm. For mid-point control $=2$, and for end point control $=1 .^{8}$ Using the emf behind leakage inductance machine equation:

$$
=\frac{l}{T}\left(\begin{array}{ll}
1
\end{array}\right)+
$$

in (14) one can obtain (after collecting terms) the following expression:

$$
\begin{aligned}
1+\left(\begin{array}{ll}
(\Delta+1) & 2
\end{array}\right)+\left(\begin{array}{ll}
1 & \Delta
\end{array}\right) & 1 \\
= & \Delta{ }_{1}^{\operatorname{ref}}+\frac{T}{l}\left(\begin{array}{l}
1
\end{array}\right)
\end{aligned}
$$

This expression can be discretised using the forward shift operator to create a discrete transfer function from the reference input to the output of the current controller:

$$
\begin{aligned}
& \left.\left(\begin{array}{ll}
2+(\Delta(+1) & 2
\end{array}\right)+\left(\begin{array}{ll}
1 & \Delta
\end{array}\right)\right) \\
& ={ }^{2} \Delta \text { ref }_{1}(1) \frac{T}{l}
\end{aligned}
$$

The at the end of this function can be considered to be a disturbance term, and can be neglected in terms of a stability analysis of the controller. ${ }^{9}$

In order to investigate the stability of the controller in relation to the accuracy of the leakage inductance estimate we can manipulate (17) into a classical transfer function form as:

$$
\begin{aligned}
() & =\frac{I()}{I^{\operatorname{ref}()}} \\
& =\frac{2 \Delta}{2+\left(\Delta \quad\left(\begin{array}{lll}
+1) & 2
\end{array}\right)+\left(\begin{array}{ll}
1 & \Delta
\end{array}\right)\right.}
\end{aligned}
$$

\footnotetext{
${ }^{7}$ It should be noted that (13) does not include the stator resistance. For PCC this is not necessary, but when used with IPC $R_{s}^{\text {est }} i \geq R_{s} i$ must be included to ensure stability of the IPC algorithm.

${ }^{8}$ Mid-point control means that the control objective is to control the current at the middle point of the control interval. This is equivalent to controlling the average current over the control interval.

${ }^{9}$ If one is considering the effects of the time varying nature of the back-emf on the performance of the controller, then this term would be important.
}

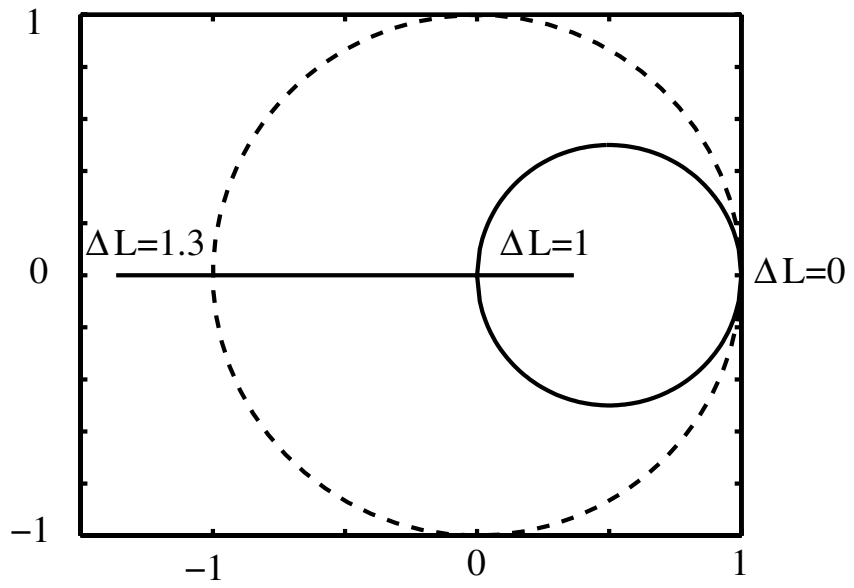

Fig. 6. Root-locus plot for PCC.

The poles of the discrete transfer function can be determined from the roots of the denominator of this expression (i.e. the characteristic equation). If the roots are inside the unit circle on the complex plane then the system is stable.

Let us consider the simplest of cases, $\Delta=1$, which implies that there is no error in the leakage inductance. It can easily be seen that the roots of the characteristic equation satisfy:

$$
(+(1))=0
$$

Clearly the roots are 1 and 0 . The first of these roots will be outside the unit circle if 2 . Therefore for mid-point control the pole is on the unit circle, and the system is marginally stable. This fact can be easily seen in simulations of the midpoint algorithm that the current can undergo large, sustained, ripple oscillations at half the control rate. One can physically see the reason for this - with this control only the mid point is constrained to the desired value, and the start and end points of the control interval can alternate between positive and negative points about this. End point control on the other hand will always be stable, offering dead-beat control.

If we consider the effect on pole locations of varying $\Delta$, for the $=1$ case, we get the root locus plot shown in Fig. 6 . As can be seen from this figure the roots of the characteristic equation say within the unit circle for $\Delta \quad 13$.

Remark 7: From Fig. 6 one can see that it is best to err on the side of underestimation of the $l$ parameter. Low values of estimated $\quad l$ will decrease the tracking performance of the algorithm, but the stability of the system will be guaranteed. $\diamond$ We can consider variations in and $\Delta$ in the magnitude plot of Fig. 7. As can be seen the magnitude of the root is greater than one for any value of $\Delta \quad 1$.

Remark 8: Note from Fig. 7 that one can also consider values of outside those that we have already considered. For example, one can have values of 1 , which means that we are controlling the current value at some point past the end of the control interval. One can achieve a larger $\Delta$ parameter tolerance by doing this, but the tracking performance of the controller will be adversely affected. $\diamond$ 


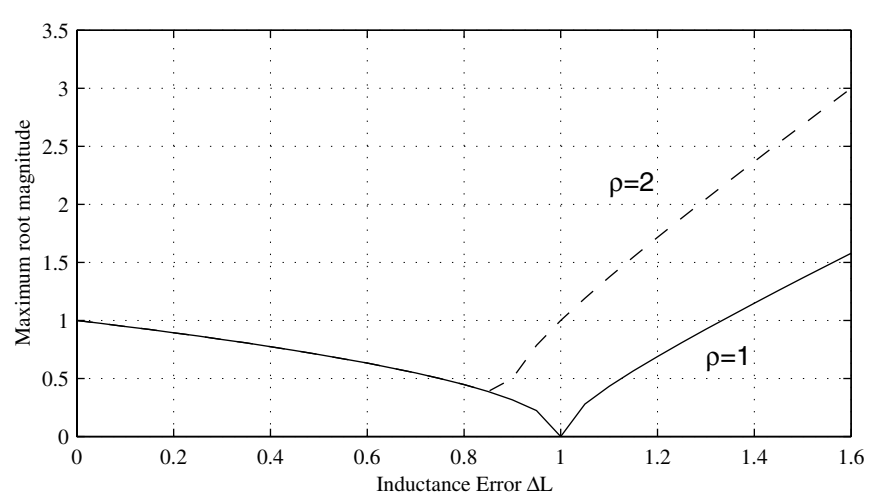

Fig. 7. Maximum root magnitude as a function of leakage inductance estimation error.

The other relevant section of the PCC is the estimation of the back-emf. One will note from (12) that under ideal conditions pred ${ }_{1}$ would be used. Clearly this is not causal. Therefore, as mentioned previously, a simple way to approximate $\quad 1$ is to use the back-emf calculated for the previous control interval (since we have all the values for (13) in this case). This technique works reasonably, but clearly there will be some errors, especially when the back-emf is rapidly varying. One obvious approach to improve this situation is the use linear extrapolation of previous back-emfs. The expressions for this are [10]:

$$
{ }_{1}^{\text {pred }}=2 \text { est } \quad \text { est }_{1}
$$

The inclusion of (21) in the controller equations will increase the order of the system dynamics. Substituting (21) into (12) and manipulating gives:

$$
1=2 \quad 1+\frac{l \Delta}{T}\left(\begin{array}{lll}
\text { ref } & (2+)+3 & 1
\end{array}\right.
$$

This equation can be used to implement the controller.

The closed loop discrete transfer function can be derived from (22):

$$
\begin{aligned}
(+\Delta(+2) & \left.3)^{2}+3(1 \quad \Delta)+\Delta \quad 1\right) \\
= & \Delta{ }_{1}^{\operatorname{ref}}(1)^{2} \frac{T}{l}
\end{aligned}
$$

Once again one can plot the root locus and root magnitudes for this transfer function. These appear in Figs. 8 and 9.

Remark 9: The first point one can see from Fig. 8 is that the range of $\Delta$ is far more limited as compared to the situation where there is no back-emf extrapolation. One can see that the range is now $05 \Delta \quad \Delta \quad 12$. Given that $l$ can change during operation (depending on the machine design), then this means that one has to have a more accurate estimate of $l$ than in the previous case. Fig. 9 further exemplifies this remark for

$=1$. One can see the larger value of the root magnitude in the extrapolation case, and the consequent smaller range for $\Delta . \diamond$

Remark 10: Whilst back-emf estimation makes the system more sensitive to accurate knowledge of ${ }_{l}$, the positive tradeoff is that the performance of the algorithm improves. The

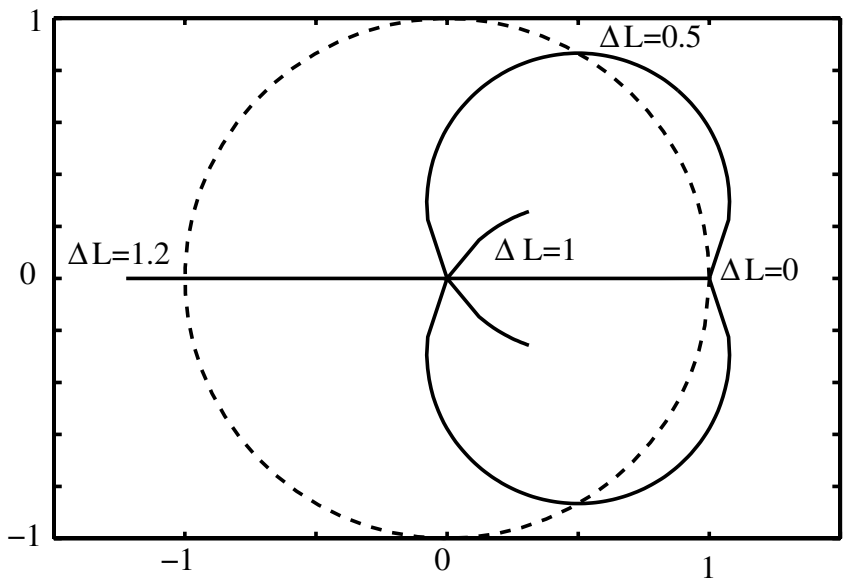

Fig. 8. Root locus for PCC with back-emf extrapolation.

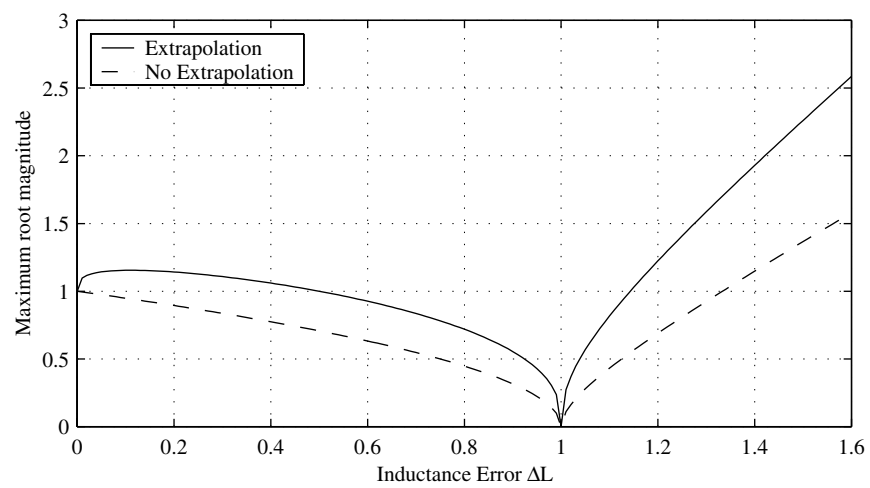

Fig. 9. Maximum root magnitude as a function of $\Delta L$ with back-emf extrapolation.

back-emf estimation offers better disturbance rejection properties. ${ }^{10} \diamond$

Remark 11: To summarise, the PCC part of the IPC control strategy is relatively insensitive to accuracy in machine model parameters. This is particularly the case when end-point control is used without back-emf estimation. $\diamond$

\section{B. Inverter Dead-time Effects}

Another important influence on the performance of the IPC algorithm is inverter dead-time. It is well known that inverter dead-time distorts the output currents of an inverter, especially near the current zero crossing points, and there have been many papers written in an effort to overcome the distortion effects [14-17]. Despite all the research, dead-time still remains as a significant contributor to PWM current waveform distortion.

Dead-time current distortion results from the fact that the voltage that is commanded by the controller is not the voltage that is actually applied to the system. In the case of the IPC algorithm, this fact can have effects that propagate through the back-emf estimator, and eventually the controller itself. Fig. 10 shows simulation results of the PCC fed with sinusoidal refer-

\footnotetext{
${ }^{10}$ More sophisticated techniques can be used with the PCC to overcome some of these problems [10].
} 


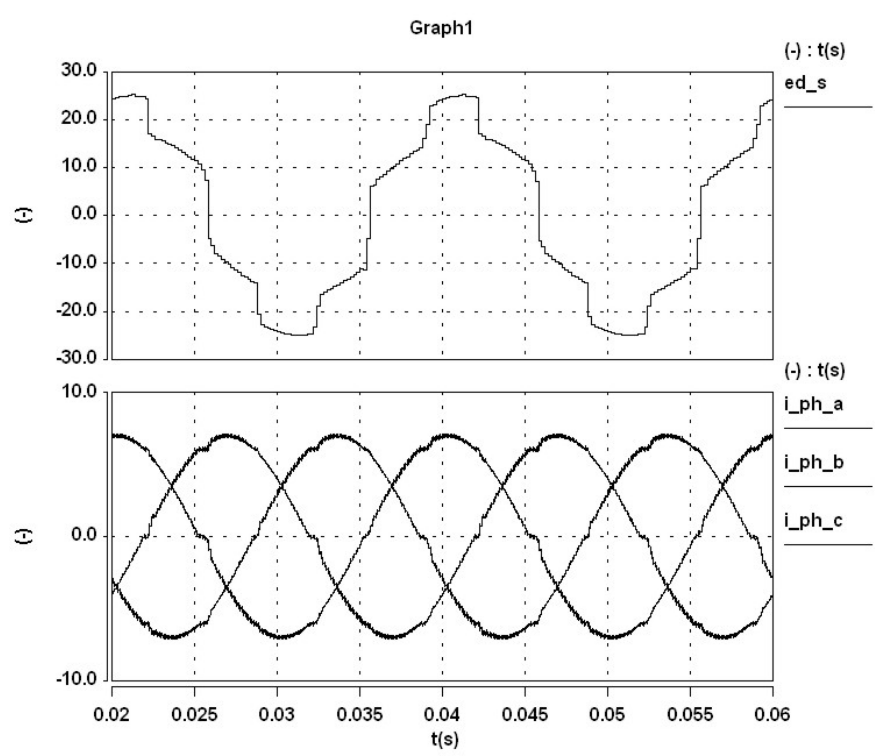

Fig. 10. Simulation of PCC with sinusoidal references and $4 \mu \mathrm{sec}$ dead-time.

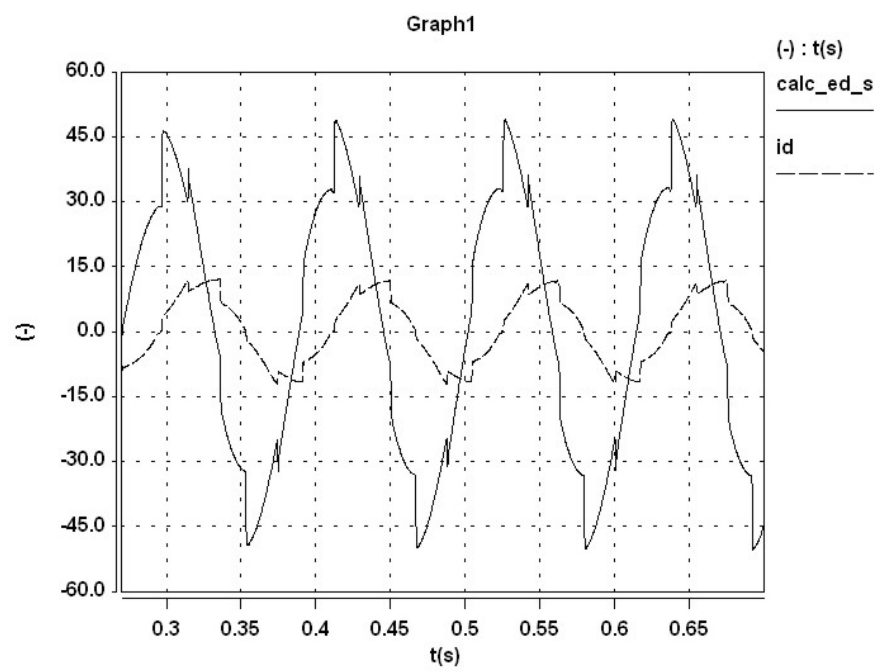

Fig. 11. The simulated $e_{d}^{\text {est }}$ voltage and $i_{d}$ current with an IPC controller with a dead-time of $4 \mu \mathrm{sec}$.

ences (i.e. there is no IPC operating). Notice the phase currents - there is the normal dead-time related distortion when the currents go through zero. This distortion is amplified and reflected in much larger distortion in the estimated back-emf.

Remark 12: Note that even though ${ }_{d}^{\text {est }}$ is very distorted in Fig. 10 the current waveforms are still following the reference with reasonable accuracy. $\diamond$

Remark 13: One can well imagine that if $\underset{d}{\text { est }}$, and the even more distorted est, are fed into (6) and (7) then distorted current references would result. These in turn are then fed back into the PCC, resulting in a substantial distortion of the currents. $\diamond$

If an IPC controlled induction machine is simulated with a 4 sec dead-time then the $d$-axis current and voltage are shown in Fig. 11.

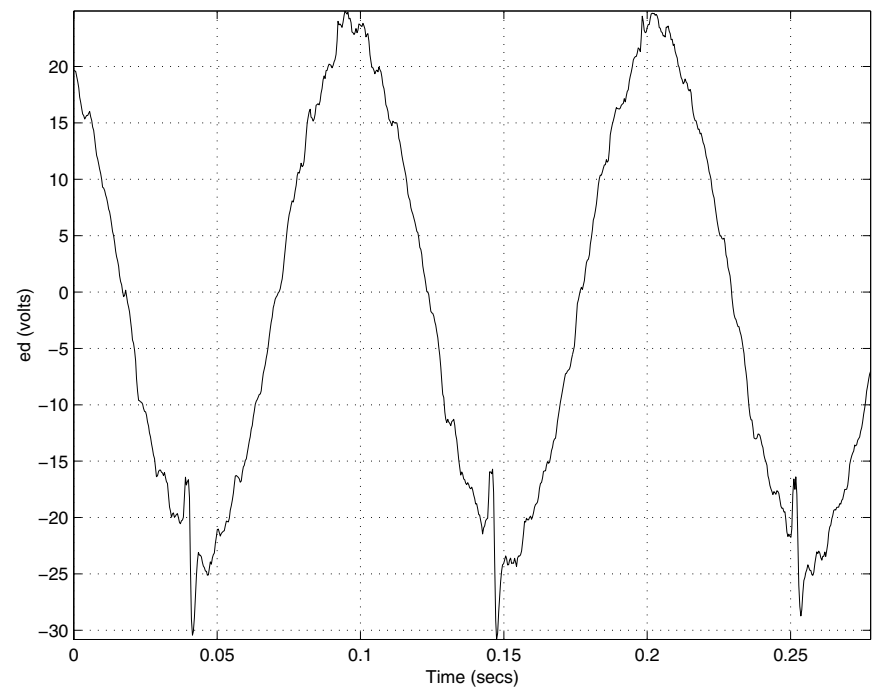

Fig. 12. Experimental results for the $e_{d}^{\text {est }}$ voltage with IPC control.

Remark 14: The ${ }_{d}^{\text {est }}$ waveform of Fig. 11 is significantly different from Fig. 10 when the power controller is not active. $\diamond$

Remark 15: One feature that one can immediately notice from Fig. 11 is that the large distortions in ${ }_{d}^{\text {est }}$ occur at the zero crossings of the $d$-axis current. Due to the frame alignment the $d$-axis current corresponds to the a-phase current. The distortion of the current is significantly more that in the case when IPC was not active. $\diamond$

Some preliminary experimental work has been carried out on IPC. Unfortunately, due to an equipment failure, more extensive experimental results cannot be shown in this paper. However, it is hoped that this will be resolved soon, and experimental results can be presented at the conference. Fig. 12 shows the experimental ${ }_{d}^{\text {est }}$ voltage under IPC control with approximately 4 sec dead-time in the inverter.

Remark 16: A comparison of Figs. 11 and 12 show the same general characteristics. The main distortions in the ${ }_{d}^{\text {est }}$ waveform of Fig. 12 occur at the current zero crossing points for the ${ }_{d}$ current, as they did in Fig. 11. $\diamond$

The distortions in the back-emf waveforms in themselves may not be a problem. However, as mentioned previously, the back-emf distortions result in significant distortions in the current waveforms, as can be seen in Fig. 11. In this figure the voltage level was low. If the voltage is increased then the amplitude of the distortions become much larger, and can result in current spikes that are high enough to cause false tripping of the drive. In addition to this problem, even if tripping is not occurring, the current spikes result in undesirable torque pulsations and performance of the drive system.

\section{B.1 A Possible Solution}

From the above discussion it is apparent the majority of the distortion in the current is due to the fact that the back-emf 
equation is using the incorrect value of in (13) ${ }^{11}$ (due to the dead-time effect). Therefore, one obvious approach is to make sure that the back-emfs are evaluated using the correct value of the applied voltage. This can be achieved by sensing the actual switching times of the inverter legs (as opposed to the commanded switching times), and then using these to evaluate the applied voltage. It should be noted that this will not eliminate dead-time distortion of the currents, but will prevent it from being amplified by the IPC loop.

An inverter system employing this approach is currently nearing completion.

\section{CONCLUSION}

This paper has outlined a new algorithm for the control of induction machines, based on manipulating the instantaneous real and imaginary power into the machine. A number of important practical issues that affect the performance of the IPC algorithm were then considered. The main conclusions that can be drawn are:

The IPC algorithm is relatively parameter insensitive compared to the FOC algorithm. This parameter insensitivity occurs in the both the reference generation and PCC sections of the algorithm.

Maximum parameter insensitivity is achieved with the PCC section of the controller if back-emf extrapolation is not used (at the price of decreased tracking performance).

Dead-time has a major influence on the performance of the IPC algorithm. If the algorithm is to work satisfactorily then dead-time at the current zero crossings must be compensated for when evaluating the machine back-emfs.

The advantages and disadvantages of IPC over other induction machine control algorithms are not clear at the moment. It is hoped that experimental studies now underway will help to resolve this issue. Analysis and simulation of IPC indicate that it appears to be very robust to inaccurate parameter knowledge.

\section{REFERENCES}

[1] F. Blaschke, "The principle of field-orientation as applied to the new transvektor closed-loop control system for rotating-field machines," Siemens Review, vol. 34, pp. 217-220, 1972.

[2] M. Depenbrock, "Direct self-control (DSC) of inverter-fed induction machine," IEEE Transactions on Power Electronics, vol. 3, pp. 420-429, Oct 1988.

[3] J. K. Kang and S. K. Sul, "New direct torque control of induction motor for minimum torque ripple and constant switching frequency," IEEE Transactions on Industry Applications, vol. 35, pp. 1076-1082, Sept/Oct 1999.

[4] H. Akagi, Y. Kanazawa, and A. Nabae, "Instantaneous reactive power compensators comprising switching devices without energy storage components," IEEE Transaction on Industry Applications, vol. IA-20, pp. 625-630, May/June 1984

[5] T. Noguchi, H. Tomiki, S. Kondo, and I. Takahashi, "Direct power control of PWM converter without power-source voltage sensors," IEEE Transactions on Industry Applications, vol. 34, pp. 473-479, May/June 1998.

[6] R. Betz and B. Cook, "Instantaneous power control - an alternative to vector and direct torque control for induction machines," Proceedings of the IEEE-IAS Annual Meeting, pp. 1640-1647, Rome, Oct. 2000.

${ }^{11}$ Remember that all the $(k+1)$ 's become $k$ 's and the $k$ 's become $(k-1)$ to maintain causality in (13).
[7] R. Betz and B. Cook, "Instantaneous power control of induction machines," Tech. Rep. EE00022, Department of Electrical and Computer Engineering, University of Newcastle, Australia, 2000. Available at: http://www.ee.newcastle.edu.au/users/staff/reb.

[8] W. Farrer and J. Miskin, "Quasi sine-wave fully regenerative inverter," Proc. Inst. Elect. Eng., vol. 120, pp. 969-976, Sept 1973.

[9] D. Holmes and D. Martin, "Implementation of a direct digital predictive current controller for single and three phase voltage source inverters," in Proceedings of the IEEE IAS-96 Annual Meeting, (San Diego), pp. 906913, IEEE, Oct 1996.

[10] S. J. Henriksen, "Digital predictive current control of induction machines," Master's thesis, Dept, Elec and Comp Eng, University of Newcastle, Australia, 2001.

[11] R. Betz, B. Cook, and S. Henriksen, "A digital current controller for three phase voltage source inverters," Proceedings of the IEEE-IAS Annual Meeting, pp. 722-729, New Orleans, Oct. 1997.

[12] S. J. Henriksen, R. E. Betz, and B. J. Cook, "Digital hardware implementation of a current controller for IM variable-speed drives," IEEE Transactions on Industry Applications, vol. 35, pp. 1021-1029, Sept/Oct 1999.

[13] R. Betz and B. Cook, "A digital current controller for three phase voltage source inverters," Jan 1997. Online: http://www.ee.newcastle.edu.au/users/staff/reb.

[14] J. W. Choi and S. K. Sul, "A new compensation strategy reducing voltage/current distortion in PWM VSI systems operating with low output voltages," IEEE Transactions on Industry Applications, vol. 31, pp. 1001-1008, Sept/Oct 1995.

[15] J. W. Choi, S. I. Yong, and S. K. Sul, "Inverter output voltage synthesis using novel dead time compensation," in IEEE Appl. Power Electronics Conference Record, pp. 100-106, 1994.

[16] Y. Murai, K. Ohashi, and I. Hosono, "New PWM method for fully digitized inverters," IEEE Transactions on Industry Applications, vol. IA-23, pp. 887-893, Sept/Oct 1987.

[17] R. P. Joshi and B. K. Bose, "Base/gate drive suppression of inactive power device of a voltage-fed inverter and precision synthesis of ac voltage and dc link current waves," in IEEE IECON Conference Record, pp. 1034-1040, 1990. 